\title{
The effect of heterogeneity for yearling weights measured in different test phases of the South African National Beef Cattle Improvement Scheme
}

\author{
R.R. van der Westhuizen ${ }^{\#}$ and T. Rust \\ ARC-Animal Improvement Institute, Private Bag X2, Irene 0062, South Africa
}

\begin{abstract}
In animal breeding, animals originating from different environments are often considered in selection decisions. Production data in these groups may differ both in mean and variance. In South Africa, yearling weights of beef cattle are recorded in two different phases of the National Beef Cattle Improvement Scheme (NBCIS). The aim of this study was to assess changes in the ranking of animals in the selection process where animals are selected on estimated breeding values (EBVs) for yearling weight before and after correcting for heterogeneity between the two phases. Yearling weights are measured in Phases B and D of the NBCIS. Yearling weights recorded in Phase D were adjusted according to the standard deviation and heritabilities of yearling weights in Phase B. To determine whether the adjustment was justified, two VCE REML analyses were performed on combined Phase B and D data to estimate breeding values. The first analysis involved unadjusted Phase D data, while the second run involved Phase D yearling weights, adjusted for heterogeneity. The ranking correlation between EBVs derived from the two runs, was $99 \%$ indicating almost no re-ranked after adjustment. It is concluded that Afrikaner yearling weights in Phase B and D can be analysed jointly without any pre-adjustments when estimating breeding values.
\end{abstract}

Keywords: Beef cattle, heritabilities, heterogeneous variances, heterogeneity, yearling weights

${ }^{\#}$ Corresponding author. E-mail: bobbie@irene.agric.za

\section{Introduction}

In animal breeding, animals originating from different environments are often considered in selection decisions. Production data in these groups may differ both in mean and in variance. Until recently, in most genetic evaluations using mixed model methodology, variances were assumed to be constant across environmental groups. However, several studies have shown that these assumptions do not hold in many cases (Hill et al., 1983; Hill, 1984; DeVeer \& Van Vleck, 1987; Boldman \& Freeman, 1990). Variances for production and conformation traits of dairy cattle have been found to be heterogeneous across herds, herdclasses, environments or other types of fixed effect levels (Dodenhoff \& Swalve, 1998). Thus, heterogeneity does not only have an effect across environmental groups, it can also have an effect on the selection of animals originating from different contemporary groups.

Heterogeneous variances have been reported for performance in beef cattle (Garrick et al., 1989). Ignoring heterogeneity may reduce the reliability of ranking and selection procedures based on Henderson's mixed model equations (Henderson, 1975), which require appropriate variance components to provide solutions with Best Linear Unbiased Prediction (BLUP) properties.

In South Africa, yearling weights in beef cattle are measured in two different phases of the National Beef Cattle Improvement Scheme (NBCIS). The objective of the present study was to evaluate the appropriateness of the multiplicative mixed model to handle data when variances are likely to be heterogeneous across herds. Re-estimation of variance components after scaling the data with the previously obtained multiplicative adjustment factors was explored to obtain a measure of the improvement in the breeding value evaluation and to detect changes in ranking of individuals across these two phases.

\section{Materials and Methods}

Afrikaner calves considered in this study participated in Phase B or Phase D of the South African NBCIS from 1982 to 1999. In Phase A, the pre-weaning growth performance of calves is evaluated. In post weaning, most of the male weaner calves complete an on-farm growth test (Phase D) or a central growth test (Phase C), while their female contemporaries as well as the remaining male weaners remain in an on-farm test up to the age of 18 months (Phase B).

In Phase B yearling weights are recorded when the calf reaches the age of $c a .12$ months. Animals in Phase D are weighed each week. Yearling weight is defined as the weight recorded at the end of the week in which the animal reaches 12 months. 
To determine the heritability for yearling weights measured in the different environments, i.e. Phase $\mathrm{B}$ and Phase D, two multitrait REML analyses were done. In the first analysis, a multitrait analysis with weaning weights and yearling weights measured in Phase B was used. The second multitrait analysis included weaning weights and yearling weights as measured in Phase D. This was done to determine to what extent the variances differ between the two environments.

The importance of non-genetic sources of variation on the traits was determined by the PROC GLM procedure of SAS (1996). Non-genetic sources that were included in the models for weaning and yearling weights were the linear and quadratic regression of the age of the calf (in days) at weaning and yearling, the linear and quadratic regression of the age of the dam and the fixed contemporary groups for weaning and yearling weights, respectively. All of the above mentioned non-genetic sources were significant $(\mathrm{P}<0.001)$ and were, therefore, included in both models.

If an attempt is made to account for heterogeneity of heritabilities across environments in an evaluation system that use a common heritability for all environments, records have to be adjusted so that the estimated phenotypic variances reflect the heterogeneity. This would guarantee a selection advantage from environments with higher heritabilities. Computationally, the least demanding approach to account for heterogeneity, is by standardizing the records, as was done by Wiggans \& VanRaden (1991), Weigel \& Lawlor (1994) and Dodenhoff \& Swalve (1998). Adjusting records so that optimum fractions, i.e., fractions maximizing response to selection, are selected from both the environments can be done as follows:

where:

$$
\mathrm{y}_{\mathrm{ij}}^{*}=\left(\mathrm{y}_{\mathrm{ji}}-\bar{y}_{\mathrm{i}}\right) \frac{\sigma_{\text {Pbase }}}{\sigma_{\mathrm{P}}} \sqrt{\frac{\mathrm{h}_{\mathrm{i}}^{2}}{\mathrm{~h}_{\text {base }}^{2}}}+\bar{y}_{\mathrm{I}}
$$

$y_{i j}^{*}=$ standardised observation of the $j^{\prime}$ th animal in environment $\mathrm{i}$;

$\mathrm{y}_{\mathrm{ji}}=$ original observation of $\mathrm{j}$ 'th animal in environment $\mathrm{i}$;

$\bar{y}_{\mathrm{i}}=$ average weight in environment $\mathrm{i}$;

$\sigma_{\text {Pbase }}=$ base phenotypic standard deviation;

$\sigma_{\mathrm{Pi}}=$ phenotypic standard deviation in environments i;

$\mathrm{h}^{2}$ base $=$ base heritability;

$\mathrm{h}^{2}{ }_{\mathrm{i}}=$ heritability in environment $\mathrm{i}$.

$\sigma_{\text {Pbase }}$ and $\mathrm{h}_{\text {base }}^{2}$ is chosen arbitrarily.

Phase D records were adjusted to meet the phenotypic variances of Phase B. As a result of the adjustment on the Phase D data, Phase D and B now have a common heritability.

In order to meet one of the objectives of this study, which was to compare the ranking of estimated breeding values (EBVs) before and after correcting for heterogeneity, two additional datasets were created. The first set was a combination of Phase B and D prior to adjustment, while the second one was a combination of Phase B and the adjusted Phase D data.

A restricted maximum likelihood analysis, fitting a multitrait animal model was used to analyse the data. The REML VCE 4.2.5 package of Groeneveld (1998) was used for this purpose. All traits were considered as traits of the calves.

The following model was used for analysis:

where:

$$
\mathrm{y}=\mathrm{Xb}+\mathrm{Z}_{1} \mathrm{a}+\mathrm{Z}_{2} \mathrm{~m}+\mathrm{e}
$$

$y=$ vector of the observations for the $i^{\text {th }}$ trait,

$b=$ vector of fixed effects for the $i^{\text {th }}$ trait,

$a=$ vector of random animal effects for the $i^{\text {th }}$ trait,

$\mathrm{m}=$ vector of random maternal effect for the $\mathrm{i}^{\text {th }}$ trait,

$\mathrm{e}=$ vector of random residual effects for the $\mathrm{i}^{\text {th }}$ trait,

$\mathrm{X}, \mathrm{Z}_{1}$ and $\mathrm{Z}_{2}$ are incidence matrixes relating records of the $\mathrm{i}^{\text {th }}$ trait to fixed and random animal effects, respectively. 


\section{Results and discussion}

After editing, there were 12416 animals that participated in Phase B with weaning and yearling weights and 2224 animals with weaning and yearling weights participating in Phase D of the NBCIS (Table 1). From Table 1 it is apparent that the standard deviation of Phase D is greater than that of Phase B. One should, however, note that the coefficient of variation is greater in Phase B. The reason for this could be that the age range of Phase B is greater than that of Phase D, and that Phase B includes both sexes where as Phase D only includes males. The standard deviation of Phase D decreases when the Phase D data are adjusted for heterogeneity between these two Phases.

Table 1 General statistics for age and weight at weaning and yearling measured in two different test phases

\begin{tabular}{lccccc}
\hline & $\mathrm{n}$ & Min. & Max. & Mean & s.d. \\
\hline Age at weaning (days) & 14640 & 150 & 270 & 210 & 28.99 \\
Weaning weight (kg) & 14673 & 95 & 280 & 178 & 29.78 \\
Phase B yearling age (days) & 12416 & 271 & 450 & 368 & 38.09 \\
Phase B yearling weight (kg) & 12416 & 118 & 373 & 221 & 46.09 \\
Phase D yearling age (days) & 2224 & 362 & 368 & 365 & 1.99 \\
Phase D yearling weight (kg) & 2224 & 156 & 450 & 302 & 50.40 \\
Adjusted Phase D yearling weight (kg) & 2224 & 193 & 412 & 300 & 38.10 \\
Phase B \& D yearling age (days) & 14640 & 271 & 450 & 366 & 35.29 \\
Phase B \& D yearling weight (kg) & 14640 & 118 & 450 & 232 & 55.33 \\
Phase B \& Adjusted Phase D & 14640 & 118 & \multirow{2}{*}{412} & 232 & 53.34 \\
Yearling weight (kg) & & & & & \\
\hline
\end{tabular}

Heritabilities and genetic correlations for weaning and yearling weights measured in two Phases of the NBCIS are presented in Table 2.

Table 2 Heritabilities (on diagonal) and genetic correlations (above diagonal) for weaning and unadjusted yearling weights measured in Phases B and D

\begin{tabular}{lcccc}
\hline & $\begin{array}{c}\text { Weaning } \\
\text { maternal }\end{array}$ & $\begin{array}{c}\text { Yearling } \\
\text { Maternal }\end{array}$ & $\begin{array}{c}\text { Weaning } \\
\text { direct }\end{array}$ & $\begin{array}{c}\text { Yearling } \\
\text { direct }\end{array}$ \\
\hline Phase B & & & & -0.19 \\
\hline Weaning maternal & 0.28 & 0.97 & -0.43 & -0.41 \\
Yearling maternal & & 0.19 & -0.61 & 0.91 \\
Weaning direct & & 0.32 & 0.28 \\
Yearling direct & & & & -0.70 \\
\hline Phase D & 0.24 & 0.96 & -0.46 & -0.76 \\
\hline Weaning maternal & & 0.25 & -0.63 & 0.91 \\
Yearling maternal & & 0.28 & 0.26 \\
Weaning direct & & & \\
Yearling direct & & & & \\
\hline
\end{tabular}

The direct heritabilities for yearling weight between the two phases are of the same order (Table 2). It was decided (because of the size of the two data sets) to adjust the Phase D yearling weight measurements with the adjustment equation mentioned previously (equations 1) to meet the standard deviation and heritability estimated for Phase B yearling weights, i.e. $\mathrm{h}_{\text {base }}^{2}=0.28$.

To determine whether the adjustment was justified, two VCE REML analyses were performed to estimate BLUP breeding values. Firstly, on the combined data set before the Phase D data was adjusted and secondly, on the combined data set after the Phase D yearling weights were adjusted for heterogeneity. Both these were multitrait analyses in conjunction with weaning weight. The results of the variance components estimated are presented in Table 3. It is apparent from these results that the heritabilities and genetic correlations between the direct and maternal heritabilities for weaning and yearling weights did not change 
markedly after adjustment. This could be the result of the vastly larger number of animals tested in Phase B (12 146) compared to the 224 animals tested in Phase D.

Table 3 Heritabilities (on diagonal) and genetic correlations (above diagonal) for weaning and yearling weights in a combined Phase B and D dataset before and after adjustments of Phase D yearling weights

\begin{tabular}{lcccc}
\hline & $\begin{array}{c}\text { Weaning } \\
\text { maternal }\end{array}$ & $\begin{array}{c}\text { Yearling } \\
\text { Maternal }\end{array}$ & $\begin{array}{c}\text { Weaning } \\
\text { direct }\end{array}$ & $\begin{array}{c}\text { Yearling } \\
\text { direct }\end{array}$ \\
\hline Before adjustment & & & & -0.16 \\
\hline Weaning maternal & 0.28 & 0.94 & -0.39 & -0.38 \\
Yearling maternal & & 0.17 & -0.56 & 0.89 \\
Weaning direct & & & 0.28 & 0.25 \\
Yearling direct & & & & -0.16 \\
\hline After adjustment & 0.28 & 0.95 & -0.40 & -0.38 \\
\hline Weaning maternal & & 0.17 & -0.58 & 0.90 \\
Yearling maternal & & & 0.29 & 0.25 \\
Weaning direct & & & & \\
Yearling direct & & & & \\
\hline
\end{tabular}

The ranking correlation between the EBVs of the two analyses was $99 \%$, indicating almost no reranking after the adjustment. This is in agreement with Reverter et al. (1997) who estimated correlations greater than 0.97 between EBVs with and without corrections for heterogeneity for different traits in Angus cattle.

\section{Conclusions}

For Afrikaner beef cattle, the effect of heterogeneous environments on selection decisions for yearling weight is small. This is probably due to the small difference between the variances estimated in the two environments. It is, therefore, concluded that yearling measurements of animals participated in Phase B and $\mathrm{D}$ can be analysed jointly without any pre-adjustments when estimating BLUP breeding values.

\section{Reference}

Boldman, K.G. \& Freeman, A.E., 1990. Adjustment for heterogeneity of variances by herd production level in dairy cow and sire evaluation. J. Dairy Sci. 73, 503-512.

DeVeer, J.C. \& Van Vleck, L.D., 1987. Genetic parameters for first lactation milk yields at three levels of herd production. J. Dairy Sci. 70, 1434-1441.

Dodenhoff, J. \& Swalve, H.H., 1998. Heterogeneity of variance across regions of northern Germany and adjustment in genetic evaluation. Livest. Prod. Sci. 53, 225-236.

Garrick, D.J., Pallak E.J., Quaas R.L. \& Van Vleck, L.D., 1989. Variance heterogeneity in direct and maternal weight traits by sex and percent purebred for Simmental-sired calves. J. Anim. Sci. 67, 25152528.

Groeneveld, E., 1998. VCE4 Version 4.2.5 User's Guide and Reference Manual, Mariensee, Germany.

Henderson, C.R., 1975. Comparison of alternative sire evaluation methods. J. Anim. Sci. 41, 760-770.

Hill, W.G., Edwards, M.R., Ahmed, M.K.A. \& Thompson, R., 1983. Heritability of milk yield and composition at different levels and variability of production. Anim. Prod. 36, 59-68.

Hill, W.G., 1984. On selection among groups with heterogeneous variance. Anim. Prod. 39, 473-477.

Reverter, A., Tier, B., Johnston, D. J. \& Graser, H.-U., 1997. Assessing the efficiency of multiplicative mixed model equations to account for heterogeneous variance across herds in carcass scan traits from beef cattle. J. Anim. Sci. 75, 1477-1485.

SAS, 1996. Statistical Analysis Systems user's guide, Release 6.12. SAS Institute Inc., Cary, North Carolina, USA.

Weigel, K.A. \& Lawlor, T.J., 1994. Adjustment for heterogeneous variance in genetic evaluations for conformation of United States Holsteins. J. Dairy Sci. 77, 1691-1701.

Wiggans, G.R. \& VanRaden, P.M., 1991. Method and effect of adjustment for heterogeneous variance. J. Dairy Sci. 74, 4350-4357. 